\title{
FIBRINA RICA EM PLAQUETAS (PRF) COMO TRATAMENTO DE COMUNICAÇÃO BUCO-SINUSAL: RELATO DE CASO
}

\section{Luiz André da Luz Silva Alves}

Acadêmico da Faculdade de Odontologia da Universidade Federal Fluminense - UFF Niterói/RJ.

\section{Fernanda Britto de Melo Silva}

Residente de Cirurgia Oral e Maxilofacial do Hospital Federal dos Servidores do Estado do Rio de Janeiro - HFSE e da Universidade Federal Fluminense - UFF, Niterói / RJ, Brasil.

\section{Caio Botelho Vieira de Lacerda}

Graduado em Odontologia pela Universidade Federal Fluminense - UFF, Niterói / RJ, Brasil.

\section{Rafael Seabra Louro}

Professor da disciplina de Cirurgia Bucal da Universidade Federal Fluminense - UFF, Niterói / RJ, Brasil.

\section{Rodrigo Figueiredo de Brito Resende}

Professor da disciplina de Cirurgia Bucal da Universidade Federal Fluminense - UFF, Niterói / RJ, Brasil.

Instituição na qual o trabalho foi realizado: Faculdade de Odontologia da Universidade Federal Fluminense - Niterói/RJ

Categoria: Relato de caso

\section{Informações do autor principal:}

Luiz André da Luz Silva Alves

Rua Alrino Sá Leitão, 24

São Gonçalo- Rio de Janeiro- RJ, Brasil.

CEP: 24743780

Email: luizandre.13@hotmail.com

Telefone: +5521980859673 


\title{
Resumo
}

As comunicações buco-sinusais são complicações pós-cirúrgicas que ocorrem mais frequentemente após exodontia de molares superiores devido a relação anatômica íntima entre o ápice das raízes desses dentes com o seio maxilar. O seu diagnóstico envolve procedimentos clínicos e radiográficos, sendo a manobra de Valsalva um passo importante do exame físico. Essas comunicações diretas entre o seio maxilar e a cavidade oral, se não tratadas de forma adequada, podem evoluir para fístulas permanentes que ocorrem quando há epitelização da mucosa. A literatura defende diferentes formas de tratamento cirúrgico, sendo a maioria através de retalhos que demandam um segundo sítio de cicatrização. Objetivando-se diminuir a morbidade desses procedimentos e a melhora da cicatrização tecidual, uma opção de tratamento é a utilização de PRF (Fibrina Rica em Plaquetas). Trata-se de uma membrana de fibrina autógena, rica em leucócitos, plaquetas e fatores de crescimento, obtida através do sangue do paciente que passa por um processo de centrifugação. O objetivo do presente trabalho é relatar um caso clínico do paciente V.A.P., leucoderma, sexo masculino, 56 anos de idade, que compareceu à Faculdade de Odontologia da Universidade Federal Fluminense, apresentando ao exame clínico e por imagem, comunicação buco - sinusal com cerca de 2,5 cm em seu maior diâmetro. Com isso, foi proposto ao paciente a ressecção da fístula e fechamento através da utilização de membranas de PRF. Após prévia utilização de antibiótico e corticoide nasal, o procedimento cirúrgico foi realizado sob anestesia local em ambiente ambulatorial, sem intercorrências e o mesmo segue em controle pós-operatório com cerca de 2 meses, com boa evolução e sem sintomatologia.

Palavras-chave: seio maxilar, comunicação buco-sinusal, fibrina rica em plaquetas.

\begin{abstract}
Buco-sinus communications are postoperative complications that occur most frequently after maxillary molar extraction due to the close anatomical relationship between the apex of the roots of these teeth and the maxillary sinus. Its diagnosis involves clinical and radiographic procedures, and the Valsalva maneuver is an important step of the physical
\end{abstract}


examination. These direct communications between the maxillary sinus and the oral cavity, if not properly treated, can progress to permanent fistulas that occur when mucosal epithelialization occurs. The literature defends different forms of surgical treatment, most of them through flaps that require a second healing site. In order to reduce the morbidity of these procedures and improve tissue healing, one treatment option is the use of PRF (Fibrin Rich Platelet). It is an autogenous fibrin membrane, rich in leukocytes, platelets and growth factors that is obtained through the patient's blood that goes through a centrifugation process. The aim of the present study is to report a clinical case of VAP patient, leucoderma, male, 56 years old, who attended the Fluminense Federal University Dental School, presenting oral and sinus communication with $2.5 \mathrm{~cm}$ at its largest diameter. Thus, the patient was proposed for resection of the fistula and closure through the use of PRF membranes. After previous use of antibiotics and nasal corticosteroids, the surgical procedure was performed under local anesthesia in an outpatient setting, uneventful and the same follows in postoperative control for 2 months, with good evolution and without symptoms.

Keywords: maxillary sinus, oral-sinus communication, platelet-rich fibrin. 


\section{Introdução}

A comunicação Buco-sinusal é descrita como o acesso direto entre cavidade bucal e o seio maxilar. É uma complicação frequente proveniente de exodontias de dentes superiores posteriores pela maior proximidade dos ápices radiculares com a região de soalho de seio maxilar. (FARIAS, J.G., ET AL, 2015; FREITAS, T.M.C., ET AL. 2003). Quando este trajeto se encontra revestido de tecido epitelial, aproximadamente a partir do terceiro dia da comunicação, denomina-se fístula (GARCIA, R.R., ET AL, 2000).

O diagnóstico de uma comunicação buco-sinusal é realizado por meio da anamnese, exame clínico e imagens. As queixas que são mais frequentemente encontradas estão relacionadas com a possível passagem de alimentos ou líquidos da cavidade bucal para o seio, timbre nasalado, halitose, coriza, dor na face ou cefaléia frontal e presença de corrimento nasal unilateral (CALVET, M.V.B., ET AL, 2014). A manobra de Valsalva é um importante instrumento para diagnóstico da fistula, afirmando que haverá saída de ar ou secreção purulenta via alveolar (FREITAS T.M.C., ET AL.2003). No exame clínico observa-se o orifício de acesso ao seio maxilar, que pode sofrer alterações de tamanho de acordo com a etiologia, sendo que aberturas de tamanho inferior à $2 \mathrm{~mm}$ tendem a se fechar espontaneamente, já quando ultrapassam os $3 \mathrm{~mm}$ tendem a permanecer (HANAZAWA Y., ET AL, 1995).

No que se refere aos exames radiográficos utilizados, pode-se citar as radiografias periapicais onde se observa a descontinuidade da linha radiopaca que delimita o assoalho do seio maxilar, porém, o uso das tomadas extra-orais, como a radiografia panorâmica e a projeção de Waters ou P.A (póstero-anterior) de face, são as técnicas que permitem melhor visualização da cavidade bucal, do seio maxilar e do trajeto da comunicação bucosinusal. Além disso, a tomografia computadorizada fornece dados como: tamanho da comunicação, características do osso e a natureza da lesão da mucosa sinusal (PARISE, G. K., ET AL, 2016). 
A melhora do reparo tecidual é uma busca constante das tecnologias aplicadas à saúde na atualidade. Na cirurgia bucomaxilofacial, a utilização dos concentrados derivados de plaquetas para aumentar o reparo dos tecidos bucais é relevante. Tais materiais são ricos em fatores de crescimento e citocinas importantes no processo inflamatório que conduz à cicatrização tecidual e a sua utilização pode acelerar este processo (EHRENFEST, D.M.D., ET AL, 2009).

Em virtude do avanço das técnicas e materiais que atuem na regeneração tecidual houve o surgimento da Fibrina Rica em Plaquetas (PRF). É obtido do sangue humano por uma técnica simples, sem manipulação bioquímica e sem a utilização de anticoagulantes. O processo se baseia na obtenção de amostras do sangue do próprio paciente, que é coletado antes da cirurgia. A partir de um processo de centrifugação obtém-se o PRF, utilizado para diversos fins na odontologia, como acelerar o processo de reparo tecidual e reduzir o desconforto pós-operatório (CHOUKROUN, J., ET AL, 2006).

Nesse contexto, o objetivo desse trabalho é relatar o caso clínico em que foram utilizadas membranas de PRF como uma opção para o fechamento de fístula bucosinusal.

\section{Considerações éticas}

Os procedimentos empregados neste trabalho seguiram os padrões éticos propostos pela Declaração de Helsinque de 1975, revisada em 2000. O paciente foi tratado na Faculdade de Odontologia da Universidade Federal Fluminense através da Clínica de dentes inclusos com os termos, de consentimento livre e esclarecido e de uso de imagens e dados para publicação científica, assinados.

O estudo foi escrito seguindo as Diretrizes do Relatório de Caso Clínico (CARE, www.care-statement.org).

\section{Relato de Caso}

Paciente V.A.P., leucoderma, sexo masculino, 56 anos de idade, procurou a Faculdade de Odontologia da Universidade Federal Fluminense (FO-UFF) com queixa principal de gosto ruim na boca e saída de líquido pelo nariz ao se alimentar, com tempo de evolução de 1 mês.

REVISTA FLUMINENSE DE ODONTOLOGIA - ANO XXVI - No 53 - Janeiro / Julho 2020 
Ao exame clínico o paciente relatava extração do segundo molar superior direito há 4 semanas e apresentava radiografia panorâmica de face realizada antes do procedimento de exodontia que indicava íntimo contato da raiz com o seio maxilar (Figura 1A). No exame intraoral, apresentava fístula buco sinusal com cerca de $10 \mathrm{~mm}$ em seu maior diâmetro com drenagem de secreção e sintomatologia compatível com Sinusite Maxilar (Figura 1B).

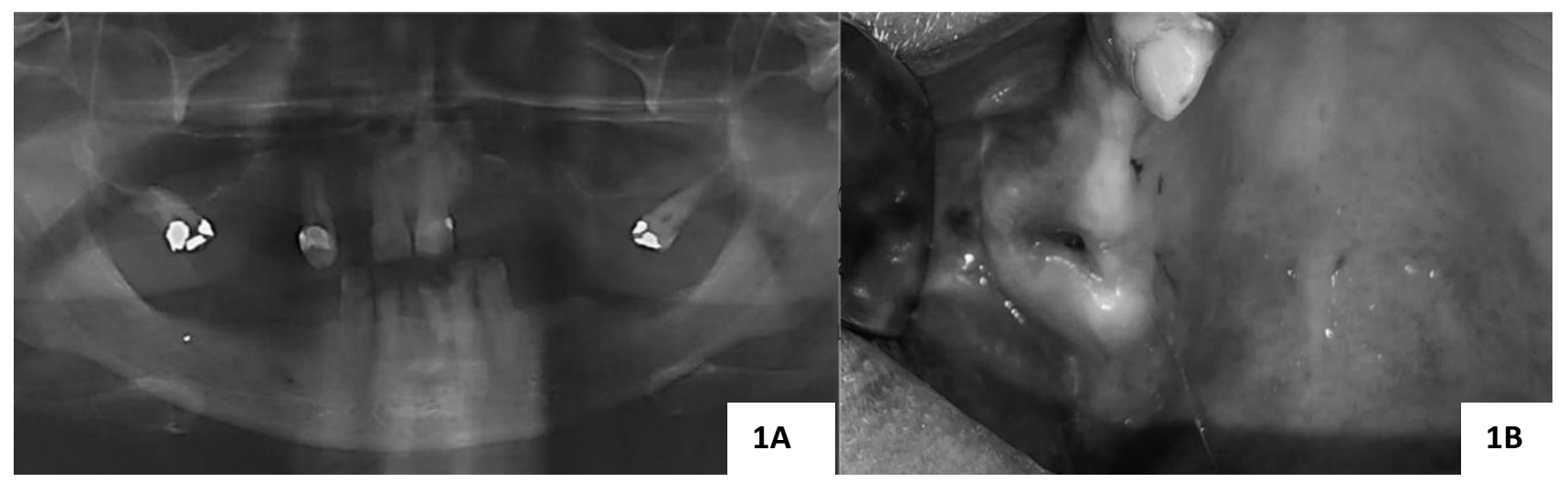

Figura 1. A) Radiografia panorâmica evidenciando íntimo contato do segundo molar superior direito com o seio maxilar; B) Visão intraoral da fístula bucosinusal de aproximadamente $10 \mathrm{~mm}$.

Com finalidade de tratar o quadro de Sinusite instalado, foi realizada antibioticoterapia com Amoxicilina 500mg. Como tratamento coadjuvante, foi prescrito Corticoide nasal Budezonida 50mg (2x ao dia) e lavagem com soro fisiológico 0,9\% no período de uma semana que antecedia o procedimento cirúrgico proposto de ressecção da fístula e fechamento com membranas de PRF.

Inicialmente, foi coletado cerca de $70 \mathrm{~mL}$ de sangue do paciente, utilizando-se o sistema fechado à vácuo de coleta, em tubos contendo sílica, preparados para acelerar o processo de coagulação da amostra. Este material foi então centrifugado seguindo o protocolo de 2700 rpm durante 12 minutos para a obtenção das membranas de PRF. 
Em seguida, o procedimento cirúrgico foi realizado em ambiente ambulatorial sob anestesia local com Lidocaína 2\% e Epirefrina 1:100.000, utilizando as técnicas de bloqueio alveolar póstero-superior e complementação palatina. A incisão foi realizada com lâmina de bisturi 15 circundando a região da fístula permitindo sua ressecção (Figura 2A e 2B).

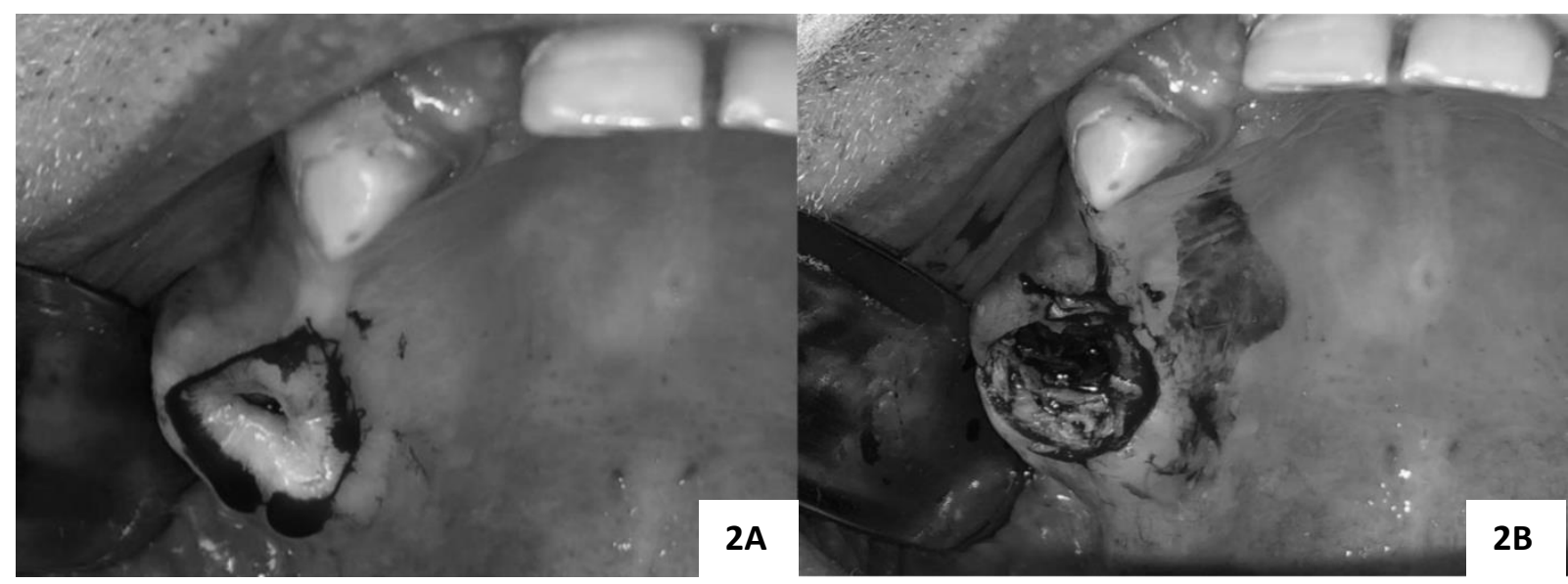

Figura 2. A) Incisão realizada contornando a área de fístula; B) Tecido ressecado evidenciando comunicação óssea com interior do seio maxilar.

Posteriormente foi feita curetagem dos tecidos com Cureta de Lucas, e irrigação com Soro Fisiológico 0,9\%. As membranas foram retiradas da centrífugas e levadas para a mesa cirúrgica, onde foram preparadas para serem utilizadas no procedimento (Figura 3A). Foram inseridas 4 membranas no interior da comunicação em direção ao seio maxilar (Figura 3B) e 4 recobrindo superficialmente a área ressecada (Figura 3C). Após revisão da hemostasia foi realizada síntese tecidual com Nylon 5.0 (Figura 3D). 


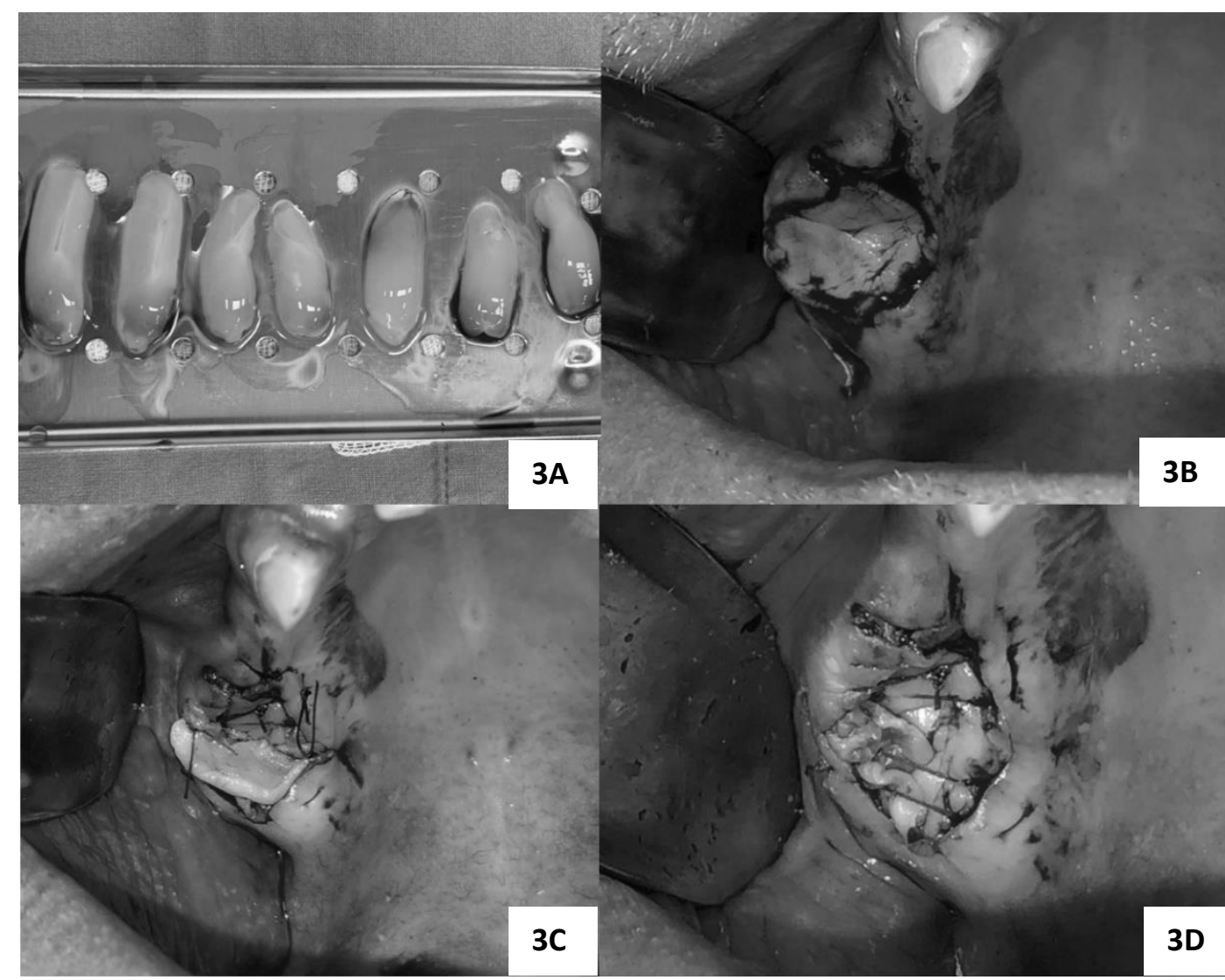

3. A) Membranas obtidas através do sangue centrifugado; B) Membranas inseridas no interior da comunicação; C) Membranas posicionadas sobre a área ressecada; D) Aspecto final com síntese tecidual.

No período pós operatório foi prescrito Amoxicilina 500mg por 7 dias, antiinflamatório não esteroidal, Nimesulida 100mg e analgésico, Dipirona 1g por 3 dias. O paciente também foi orientado do uso da placa de acetato.

Durante o acompanhamento pós-operatório imediato, o paciente apresentou boa evolução, sem complicações na ferida cirúrgica e com resolução das queixas prévias. Em acompanhamento de 1 mês, ao exame clínico o paciente segue sem alterações ou sintomatologia.

\section{Discussão}


As comunicações buco-sinusais frequentemente ocorrem como resultado da exodontia de dentes superiores posteriores devido à sua proximidade com o seio maxilar. Existem, porém, outros fatores etiológicos menos frequentes como traumatismo gerado pelo uso inadequado de instrumentos, destruição do seio maxilar por lesões periapicais e remoção de cistos e/ou tumores do palato ou do seio maxilar (PARISE, G. K., ET AL, 2016).

Diversos são os tratamentos propostos para a correção, todos visando promover o permanente fechamento da comunicação, além da eliminação da doença sinusal. A escolha da técnica é bastante controversa, contudo, todos os autores concordam que deve se esperar a eliminação da infecção do antro, antes que se proceda qualquer tipo de tentativa de fechamento. Autores afirmam que o fechamento primário, em até 48 horas, tem elevados índices de sucesso (90 a 95\%), sendo que esta taxa cai para $67 \%$ quando o fechamento se dá secundariamente.

O fechamento primário pode ocorrer espontaneamente quando os defeitos são menores que $2 \mathrm{~mm}$ de diâmetro, devendo-se somente estabilizar o coágulo com suturas que reposicionem o tecido, mantendo compressa com gaze de 1 a 2 horas sobre a sutura. Caso esse fechamento primário não venha a ter sucesso ou a comunicação seja maior ou igual a $3 \mathrm{~mm}$, podemos lançar mão de diversas técnicas cirúrgicas. (CALVET, M.V.B., ET AL, 2014).

O Retalho Palatino rodado é recomendado para o fechamento tardio de fístulas buco-sinusais, especialmente nos casos em que o fechamento com retalho vestibular tenha falhado. Suas desvantagens são citadas como sendo difícil a rotação do retalho palatino, possibilidade de necrose tecidual, hemorragia da artéria palatina maior e desconforto aos pacientes devido à área cruenta (PARISE, G. K., ET AL, 2016). Em contrapartida, autores defendem o uso da rotação de retalhos palatinos e justificam sua preferência alegando ser um retalho espesso, com um ótimo suprimento sanguíneo, levando ao sucesso e evitando-se a necrose tecidual. (FREITAS T.M.C., ET AL, 2003).

O retalho vestibular deslizante, é um tipo de retalho que pode resultar em um fundo de sulco vestibular muito superficial, o que pode interferir com a reabilitação e na 
manutenção da higiene protética. Embora apresente bom suprimento sanguíneo, esses retalhos exigem manipulação cuidadosa (FREITAS T.M.C., ET AL, 2003).

Alguns autores preferem o retalho bucal deslizante por considerem um tecido de fácil distensão, fácil execução. No entanto, apresenta como desvantagens: a perda considerável de fundo de vestíbulo, necessidade de pedículos amplos, possíveis infecções e hematomas, e, mais raramente, injúrias ao nervo facial e ducto parotídeo (CALVET, M.V.B., ET AL, 2014).

As técnicas de enxerto de osso para o tratamento de comunicações buco-sinusais grandes ou moderadas demonstram ser inovadoras, bem-sucedidas e previsíveis, e permitem evitar os efeitos colaterais clínicos, como a morbidade do local doador, relacionados com os retalhos de tecido mole. (PARISE, G. K., ET AL, 2016).

A bola adiposa de Bichat ou corpo adiposo bucal quando utilizada para o fechamento de comunicações se apresenta como um procedimento simples e rápido, trazendo mínimo desconforto ao paciente, preservando a profundidade de sulco e com baixa morbidade. Entretanto, deve-se citar as desvantagens da técnica, que está sujeita a falhas de integração por necrose ou infecção, incidência de trismo no pós-operatório assim como de retração ou deiscência do enxerto. (FARIAS J.G., ET AL, 2015).

Algumas vantagens são relatadas na literatura relacionada com a utilização de PRF para fechamento de comunicação bucosinusal, tal como a sua preparação, é uma técnica simples, eficaz e de baixo custo. Quando utilizada como uma membrana, evita-se um procedimento cirúrgico em um segundo sítio e resulta numa redução no desconforto do paciente durante o período de pós-operatório. (CHOUKROUN, J., ET AL, 2006).

\section{Conclusão}

A utilização de membranas de fibrina obtidas através do sangue do próprio paciente mostrou-se um tratamento eficaz por não apresentar recidiva da lesão e ainda se mostrar como uma alternativa de baixo custo. Além disso, não é necessário a intervenção em um segundo sítio cirúrgico para utilizar retalho no fechamento, o que diminui consideravelmente a morbidade do procedimento.

REVISTA FLUMINENSE DE ODONTOLOGIA - ANO XXVI - N 53 - Janeiro / Julho 2020 


\section{Referências Bibliográficas:}

1. Farias, J.G; Câncio, A.V.; Barros, L.F. Fechamento de fistula buco sinusal utilizando o corpo adiposo bucal - Técnicas convencional x Técnica do túnel Relato de casos clínicos. Disponível em: http://revodonto.bvsalud.org/scielo.php?pid= S1808-52102015000300005 \&script=sci_arttext\&tlng=pt. Rev. cir. traumatol. buco-maxilo-fac. vol.15 no.3 Camaragibe Jul./Set. 2015.

2. Freitas, T.M.C., Farias, G. J. Mendonça G. R., Alves F.M., R.P. R Jr., Câncio A.V. Fístulas oroantrais: diagnóstico e propostas de tratamento. Disponível em: http://www.scielo.br/scielo.php?pid=S0034-7 2992003000600018 script=sci_ abstract tlng=pt. Rev Bras Otorrinolaringol. V.69, n.6, 838-44, nov./dez. 2003.

3. Garcia, R.R.; Rabêlo L.R.S.; Moraes M.; Moreira R.W.F.; Albergaria-Barbosa J.R. Utilização de enxerto pediculado do corpo adiposo da bochecha no tratamento de comunicações oro-antrais. Rev Port Estomatol Cir Maxilofac 2000; 41: 17-24.

4. Calvet, M.V.B; Castro, B.R.A.; Agostinho, C.N.L.F.; Bastos, E.G. Fechamento de comunicação buco-antral com bola adiposa de bichat: revisão de literatura e relato de caso. Rev. Ciênc. Saúde, São Luís, v.16 n.2, p. 106-111, jul-dez, 2014.

5. Hanazawa Y.; Itoh K.; Mabashi T.; Sato K. Closure of oroantral communications using a pedicled buccal fat pad graft. Disponível em: https://www.joms.org/article/0278-2391(95)9 0329-1/pdf. July 1995 Volume 53, Issue 7, Pages 771-775.

6. Parise, G. K.; Tassara, L. F. R. Tratamento cirúrgico e medicamentoso das comunicações buco-sinusais: uma revisão da literatura. PERSPECTIVA, Erechim. v. 40, n. 149, p. 153-162, 2016.

7. Ehrenfest, D. M. D.; Rassmund, L.; Albrektsson, T. Classification of platelet concentrates: from pure platelet-rich plasma (P-PRP) to leucocyte and plateletrich fibrin (L- PRF). Trends in Biotechnology, v.27, n.3, p.158-167, 2009. 
8. Choukroun, J.; Diss, A.; Simonpieri, A.; Girard, M.O.; Schoeffler, C.; Dohan, S.L.; et al. Platelet- rich fibrin (PRF): A second-generation platelet concentrate. Part V: Histologic evaluations of PRF effects on bone all o graft maturation in sinus lift. Oral Surg Oral Med Oral Pathol Oral EndodRadiol 2006; 101: 299303. 